\title{
Selective Alterations of White Matter Associated with Visuospatial and Sensorimotor Dysfunction in Turner Syndrome
}

\author{
Marie Holzapfel, Naama Barnea-Goraly, Mark A. Eckert, Shelli R. Kesler, and Allan L. Reiss \\ Center for Interdisciplinary Brain Sciences Research, Department of Psychiatry and Behavioral Sciences, Stanford University School of Medicine, Stanford, \\ California 94305
}

\begin{abstract}
Turner syndrome (TS) is a neurogenetic disorder characterized by impaired spatial, numerical, and motor functioning but relatively spared verbal ability. Results from previous neuroimaging studies suggest that gray matter alterations in parietal and frontal regions may contribute to atypical visuospatial and executive functioning in TS. Recent findings in TS also indicate variations in the shape of parietal gyri and white matter microstructural anomalies of the temporal lobe. Diffusion tensor imaging and structural imaging methods were used to determine whether 10 females with TS and 10 age- and gender-matched control subjects exhibited differences in fractional anisotropy, white matter density, and local brain shape. Relative to controls, females with TS had lower fractional anisotropy (FA) values in the deep white matter of the left parietal-occipital region extending anteriorly along the superior longitudinal fasciculus into the deep white matter of the frontal lobe. In addition, decreased FA values were located bilaterally in the internal capsule extending into the globus pallidus and in the right prefrontal region. Voxel-based morphometry (VBM) analysis showed corresponding white matter density differences in the internal capsules and left centrum semiovale. Tensor-based morphometry analysis indicated that the FA and VBM results were not attributable to differences in the local shape of brain structures. Compared with controls, females with TS had increases in FA values and white matter density in language-related areas of the inferior parietal and temporal lobes. These complementary analyses provide evidence for alterations in white matter pathways that subserve affected and preserved cognitive functions in TS.
\end{abstract}

Key words: DTI; MRI; voxel-based morphometry; Turner syndrome; visual spatial; white matter; sensory motor

\section{Introduction}

Turner syndrome (TS), which results from the complete or partial absence of an X chromosome in phenotypic females, occurs in $\sim 1$ in 2000 live births (Lippe, 1991). The TS phenotype is characterized by short stature, gonadal dysgenesis, and a cognitive profile of relative strengths in verbal skills (Ross et al., 2000) with impairments in visuospatial and arithmetic processing (Waber, 1979; Pennington et al., 1985; Bender et al., 1993; Murphy et al., 1994; Rovet and Ireland, 1994), motor function (Ross et al., 1996), executive function (McCauley et al., 1987), and social cognition (Ross et al., 2000). Individual variation in the physical and cognitive characteristics in TS are believed to be attributable to a number of factors, including mosaic karyotypes consisting of partial X or $\mathrm{Y}$ chromosome fragments and possibly X-linked imprinting (Skuse et al.,

Received Feb. 28, 2005; accepted May 10, 2006.

This work was supported by National Institutes of Health Grants HD031715 and MH050047 and the Sinclair Research Training Fund. We appreciate the support of our colleagues at the Lucas Center for Magnetic Resonance Imaging and Spectroscopy, in particular, Dr. Roland Bammer for his help with acquisition and analysis of diffusion weighted images.

Correspondence should be addressed to Dr. Allan L. Reiss, Department of Psychiatry and Behavioral Sciences, Stanford University School of Medicine, 401 Quarry Road, Stanford, CA 94305-5719. E-mail: reiss@stanford.edu. DOI:10.1523/JNEUROSCI.1764-06.2006

Copyright $\odot 2006$ Society for Neuroscience $\quad$ 0270-6474/06/267007-07\$15.00/0
1997; Kesler et al., 2003). These factors (Murphy et al., 1993) may result in altered neurodevelopment and impaired cognitive ability.

Functional magnetic resonance imaging (fMRI) studies indicate that visuospatial and arithmetic processing deficiencies in TS may be associated with aberrant function in the frontoparietal systems (Kesler et al., 2004b), whereas deficits in executive and storage/retrieval operations may be associated with dysfunction in the inferior parietal lobe, dorsolateral prefrontal cortex, and caudate (Haberecht et al., 2001). Two positron emission tomography studies have found decreased glucose metabolism in parietal-occipital regions and in the temporal cortex and insula in TS (Clark et al., 1990; Murphy et al., 1997). These functional imaging studies suggest that deficits in tasks of visuospatial working memory and visuospatial orientation processing may arise from impaired frontoparietal connections (Haberecht et al., 2001; Tamm et al., 2003).

Complementing the findings of atypical cerebral functioning seen in fMRI studies, neuroanatomic studies of TS have consistently found decreased gray matter volume in the bilateral parietal lobes, parieto-occipital region, and subcortical gray matter (Murphy et al., 1993; Reiss et al., 1995; Brown et al., 2002; Good et al., 2003; Kesler et al., 2003; Molko et al., 2003), as well as increased volume of the superior temporal gyrus 
(STG) (Kesler et al., 2003), amygdala (Kesler et al., 2004a,b), and orbitofrontal cortex (Good et al., 2003). Recent volumetric MRI localized parietal lobe findings to the bilateral superior parietal lobule and postcentral gyrus (Brown et al., 2004). Whole-brain analyses of white matter volume in TS have revealed decreased white matter in the bilateral occipital lobe (Brown et al., 2002). To date, only one study has specifically focused on white matter morphology in TS. Molko et al. used high-resolution anatomical, diffusion tensor images and sulcal morphometry to analyze 14 adult subjects with varying karyotypes associated with TS. They found structural changes in the bilateral superior temporal sulcus, orbitofrontal cortex, right centrum semiovale, and right intraparietal sulcus (Molko et al., 2004). However, this study included participants with mosaic genotypes, which can confound between group differences (Zinn et al., 1998) and did not consider the potential effects of the intelligence quotient (IQ).

The current study included only nonmosaic, monosomic (45X) children, adolescents, and young adults with TS. We used complementary imaging methods to investigate whole-brain white matter anisotropy and concentration in TS: diffusion tensor imaging voxel-based morphometry (DTI-VBM), voxel-based morphometry (VBM), and tensor-based morphometry (TBM). Based on the neurocognitive profile of TS and previous neuroimaging findings from our group and others, we hypothesized that white matter structure in TS would be most adversely affected in regions contributing to spatial/perceptual cognition and visuospatial working memory, such as parieto-occipital, frontoparietal, and frontostriatal pathways. We also predicted that white matter anatomy would be most "preserved" in regions associated with the relatively strong verbal abilities in this condition, particularly in the perisylvian language region.

\section{Materials and Methods \\ Overview}

In the current study, we used DTI data to perform DTI-VBM analysis, and high-resolution anatomical [spoiled gradient-recalled echo (SPGR)] data to perform the VBM and TBM analyses. DTI is a magnetic resonance-based imaging method that allows visualization of molecular movement in vivo and subsequent characterization of this movement in three-dimensional space. In brain white matter tracts, molecular movement is highly restricted, mostly by neuronal sheathes and myelin. As a result, water movement along one axis of an axon is greater than perpendicular to it. Using DTI, one can assess this restricted movement and thus visualize the structure and direction of axons. In this study, we used fractional anisotropy (FA), a commonly used intravoxel scalar that reflects the degree of diffusion anisotropy (directionality) within a voxel. Anisotropy within white matter is determined mostly by microstructural features of the tissue, including fiber diameter and density, degree of myelination (Basser, 1995), and macrostructural features such as intravoxel fiber-tract coherence (Basser and Pierpaoli, 1996).

VBM involves a voxelwise comparison of the local concentration of gray or white matter between two groups of subjects. Finally, TBM is a method of identifying regional structural differences from the gradients of deformations fields.

\section{Subjects}

Participants included 10 young females with TS and 10 typically developing control female subjects individually matched for age. Subjects ranged in age from 7.1 to 24.4 years (group with TS mean age, $16 \pm 5.12$; control mean age, $15.31 \pm 4.58$ ). All subjects were right handed.

Individuals with TS were recruited through the National Turner Syndrome Society, local physicians, and the Center for Interdisciplinary Brain Sciences Research (CIBSR) website (http://cibsr.stanford.edu). All families were screened over the telephone regarding karyotype results, cognitive abilities, and physical impairments. Only subjects with IQ above 70 and a cytogenic status of nonmosaic 45X, confirmed from a standard karyotype assessment, were included in this study. The hormone replacement status of all was known, with all but two having received growth hormone and five receiving cyclins for menstruation and development. Control subjects were recruited through advertisements in local newspapers and parent networks. A minimum full-scale IQ of 85 and absence of previous neurological or psychiatric disorder were used as inclusion criteria for control subjects. There was no significant difference in verbal IQ between the two groups (TS mean, $111 \pm 19.8$; control mean, $113.7 \pm 11.5$; $p=0.715)$. As expected, there was a significant difference in performance IQ between the groups (TS mean, $96.9 \pm 12.7$; control mean, $114.4 \pm 13.1 ; p=$ $.007)$. After completely describing the study protocol to all participants and their parents, we obtained written informed consent as approved by the Institutional Review Board of Stanford University.

\section{MRI protocol}

Magnetic resonance images of each subject's brain were acquired using a 1.5 T whole-body GE-Signa Horizon scanner (GE Medical Systems, Milwaukee, WI). A DTI sequence was based on a single-shot spinecho echo-planar imaging sequence with diffusion sensitizing gradients applied on either side of the $180^{\circ}$ refocusing pulse (Moseley, 1991; Basser et al., 1994). Imaging parameters for the diffusionweighted sequence were as follows: field of view (FOV), $24 \mathrm{~cm}$; matrix size, $128 \times 128$ ( 19 slices) or $128 \times 128$ zero filled to $256 \times 256(18$ slices); echo time (TE), $106 \mathrm{~ms}$; repetition time (TR), $6000 \mathrm{~ms}$; 18 or 19 axial-oblique slices; slice thickness, $5 \mathrm{~mm}$ /skip $1 \mathrm{~mm}$. The scan was prescribed from the top of the brain and included only the most superior part of the cerebellum. Diffusion gradient duration was $\delta=$ $32 \mathrm{~ms}$, and diffusion weighting was $b=900 \mathrm{~s} / \mathrm{mm}^{2}$. In addition, two reference measurements ( $b 0$ scans) were performed and averaged for each slice after removing the diffusion sensitizing gradients. Diffusion was measured along six noncollinear directions: $\mathrm{XY}, \mathrm{XZ}, \mathrm{YZ},-\mathrm{XY}$, $-\mathrm{XZ}$, and $-\mathrm{YZ}$. This pattern was repeated four times for each slice, with the sign of all diffusion gradients inverted for odd repetitions.

To aid in the localization of white matter differences, a threedimensional, high-resolution T1-weighted anatomic gradient and a receptive field-SPGR, MRI sequence with the following parameters was used: TR, $35 \mathrm{~ms}$; TE, $6 \mathrm{~ms}$; flip angle, $45^{\circ}$; number of excitations, 1 ; matrix size, $256 \times 256$; FOV $24 \mathrm{~cm}^{2}$; for 124 contiguous slices of $1.5 \mathrm{~mm}$ width.

\section{Image processing}

DTI. Eddy current effects in the diffusion-weighted images (i.e., geometric distortions that vary from one diffusion direction to the next) were unwarped before averaging (de Crespigny and Moseley, 1998). Averaging of the four magnitude images effectively removed the effect of gradient cross-terms between the diffusion sensitizing and imaging gradients.

The FA was calculated for each voxel according to Basser and Pierpaoli (1996) to produce a fractional anisotropy image. The FA images were further processed using Statistical Parametric Mapping software (SPM99; Wellcome UK, London, UK). The T2-weighted image template from the Montreal Neurological Institute (MNI) was used to determine normalizing parameters subsequently applied, using affine transformation, to the FA images using SPM99. A template comprising adult brains was used for normalization based on previous studies showing that normalization of pediatric brain scans to adult templates can be accomplished relatively well, especially when subjects are above 6 years of age (Muzik et al., 2000). Normalized FA images were then smoothed with a $4 \mathrm{~mm}$ kernel.

The kernel choice for the DTI-VBM $(4 \mathrm{~mm})$ is similar to that used in a previous DTI-VBM study of TS, thus facilitating comparison with existing results (Molko et al., 2004). However, as pointed out in a recent study (Jones et al., 2005), there is no standard for smoothing kernel dimensions in the field of DTI-VBM.

A white matter mask derived from the averaged, normalized SPGR image was used to restrict the analysis to the white matter tracts and eliminate noise and edge effects of the T2 images. The mask excluded the cerebellum and the brainstem, which were not completely scanned in the 
Table 1. Clusters of significant between group differences in fractional anisotropy and voxel-based morphometry

\begin{tabular}{|c|c|c|c|c|c|c|c|c|}
\hline \multicolumn{4}{|l|}{$\overline{\mathrm{DTI}}$} & \multicolumn{4}{|l|}{ VBM } & \multirow{2}{*}{$\begin{array}{l}\text { TBM } \\
\text { Confirmatory } \\
\text { TBM analysis }\end{array}$} \\
\hline Location of significant FA differences & $\begin{array}{l}\text { Cluster size } \\
\text { in voxels }\end{array}$ & $\begin{array}{l}\text { Talairach coordinates of } \\
\text { most significant voxel } \\
(x, y, z)\end{array}$ & Zscore & $\begin{array}{l}\text { Location of differences } \\
\text { between groups in VBM }\end{array}$ & $\begin{array}{l}\text { Cluster size } \\
\text { in voxels }\end{array}$ & $\begin{array}{l}\text { Talairach coordinates of } \\
\text { most significant voxel } \\
(x, y, z)\end{array}$ & Zscore & \\
\hline \multicolumn{9}{|l|}{ Turner group $<$ control group } \\
\hline Left internal capsule and globus pallidus & 1502 & $-20,-5,9$ & 4.94 & $\begin{array}{l}\text { Left internal capsule } \\
\text { Superior temporal gyrus }\end{array}$ & 654 & $-24,-1,15$ & 5.38 & NS \\
\hline & & & & & 162 & $-48,-36,11$ & 3.38 & \\
\hline Right internal capsule & 634 & $20,-8,-8$ & 4.45 & Right internal capsule & 308 & $24,-2,15$ & 3.70 & NS \\
\hline $\begin{array}{l}\text { forward along the superior longitudi- } \\
\text { nal fasciculus into the frontal lobe }\end{array}$ & 864 & $-22,-47,32$ & 4.04 & Left superior frontal gyrus & 49 & $-14,-9,50$ & 3.56 & NS \\
\hline $\begin{array}{l}\text { Right prefrontal lobe, near the head of } \\
\text { the caudate and orbitofrontal cortex }\end{array}$ & 313 & $22,23,-10$ & 3.91 & 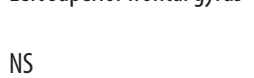 & & & & NS \\
\hline \multicolumn{9}{|l|}{ Turner group > control group } \\
\hline $\begin{array}{l}\text { Left temporal lobe, at the junction of } \\
\text { middle, superior, and inferior gyri }\end{array}$ & 463 & $-44,-56,5$ & 5.97 & $\begin{array}{l}\text { Left inferior/ middle } \\
\text { temporal gyri }\end{array}$ & $\begin{array}{l}69 \\
28\end{array}$ & $\begin{array}{l}-54,-64,-2 \\
-55,-51,-10\end{array}$ & $\begin{array}{l}3.03 \\
2.07\end{array}$ & NS \\
\hline $\begin{array}{l}\text { Right centrum semiovale, base of the } \\
\text { precentral and middle frontal gyri }\end{array}$ & 425 & $33,0,31$ & 3.98 & NS & & & & NS \\
\hline Left temporoparietal junction & 391 & $-32,-46,19$ & 3.79 & $\begin{array}{l}\text { Inferior parietal lobule } \\
\text { Temporoparietal junction }\end{array}$ & $\begin{array}{l}124 \\
26\end{array}$ & $\begin{array}{l}-34,-43,39 \\
-46,-61,20\end{array}$ & $\begin{array}{l}2.72 \\
2.34\end{array}$ & NS \\
\hline
\end{tabular}

Results are shown with their peak coordinates in Talariach space and the associated Z scores, along with their size in voxels

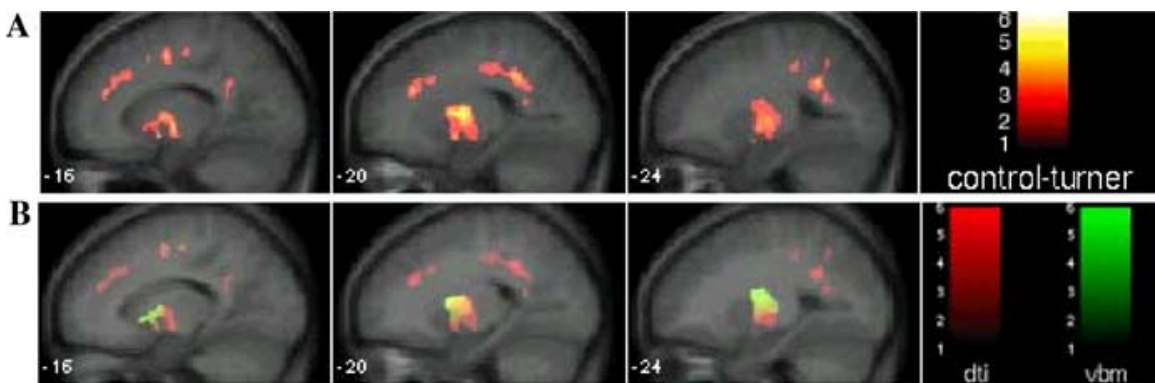

Figure 1. $\boldsymbol{A}$, Voxels that showed significant reduction in white matter fractional anisotropy in TS subjects compared with controls, mapped onto an average T1-weighted image of control and Turner brains. $\boldsymbol{B}$, Regions in which reduced white matter density overlapped with reduced FA in TS subjects are shown in yellow (superimposed on $\boldsymbol{A}$ ).

sequence prescribed. Smoothed images for controls and subjects with TS were compared using voxelwise two-tailed $t$ tests. Results were normalized to $Z$ scores to provide a statistical measure of differences between voxels that are independent of sample size. Finally, the joint expected probability distribution of the height and extent of $Z$ scores, with height $(Z>1.72 ; p<0.05)$ and extent $(p<0.05)$ thresholds, was used to determine the presence of significant clusters of difference and correct for spatial correlation in the data (Poline et al., 1997).

VBM. This technique was used primarily to determine whether white matter density differences were present within significant FA clusters (Ashburner and Friston, 2000). VBM processing was performed using the same normalization steps that were used for the diffusion tensor image processing to maintain consistency across the techniques and to ensure that the spatial dimensions of the FA and structural images were equivalent. The SPGR images were normalized using the MNI T1-weighted image template and visually inspected to ensure they were free of normalization errors. The normalized isotropic images were then segmented to obtain white matter images that were smoothed using a $10 \mathrm{~mm}$ Gaussian kernel. Traditional VBM processing (Ashburner and Friston, 2000), rather than optimized VBM (Good et al., 2002), was performed so that explicit masking could be performed to compare white matter density within significant FA clusters. This procedure provided additional information as to whether the FA results were related to the amount of white matter or specific differences in fiber orientation. The statistical process used for the DTI data were also used for the VBM analysis.
TBM. This final method for image analysis was used to detect differences in local brain shape. The parameters used to normalize each image to a template brain indicate the direction and how much each voxel nonlinearly deformed to fit to a template image. Examination of the nonlinear deformations, in the form of Jacobian determinants, provides information about local shape and can be used to indicate whether the groups differ in local brain shape. In this study, TBM was used to determine whether areas of FA difference could be attributed to previously reported differences in TS brain shape (Molko et al., 2003, 2004). The deformation fields acquired from the nonlinear registration of brain images during normalization were processed using SPM99 (Wellcome UK).

\section{Results}

Results of the DTI, VBM, and TBM analyses are presented in Table 1 and Figures 1-3 (see also http://cibsr.stanford. edu/publications/abstracts/TSVision05.htm and the supplemental movie, available at www.jneurosci.org as supplemental material).

\section{Frontoparietal and parieto-occipital pathways}

Subjects with TS had significantly decreased FA values compared with controls in white matter of the left parietal-occipital region that extended anteriorly throughout the superior longitudinal fasciculus (SLF) and into the deep white matter of the frontal lobe (Fig. 1). A small but significant region of decreased white matter density was observed within this cluster at the base of the left precentral gyrus. There were no significant shape differences between groups in this cluster.

Subjects with TS also had significantly decreased FA compared with controls in the right prefrontal cortex, with the maximum peak located adjacent to the caudate and extending into the orbitofrontal region. There were no significant white matter density or shape differences between the groups in this cluster. 

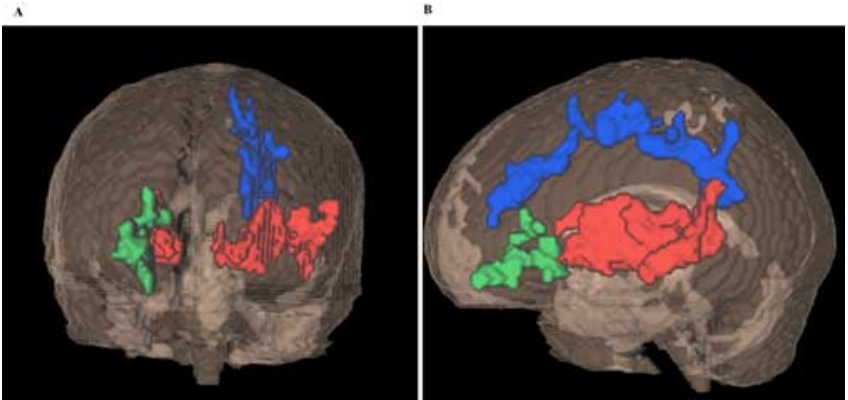

Figure 2. A three-dimensional representation of aberrant white matter tracts in TS (left frontoparietal cluster is shown in blue; right prefrontal cluster shown in green; bilateral internal capsule cluster is shown in red), as derived from FA maps positioned within a transparent, volumetrically rendered average image of all TS subjects: $\boldsymbol{A}$, anterior view; $\boldsymbol{B}$, sagittal view.

\section{Internal capsule pathways}

Subjects with TS exhibited significantly decreased FA and white matter density within the right and left internal capsule, extending throughout the anterior and posterior limbs adjacent to the globus pallidus (Fig. 2). There were no significant shape differences between groups in this cluster.

Results of the reverse comparison (increased FA values in TS when compared with controls) revealed significant differences in sensorimotor regions located in the white matter of the right centrum semiovale, at the base of the right precentral gyrus, and extending anteriorly into the middle frontal gyrus (MFG) (Table 1). There were no white matter density differences between the groups within this FA voxel cluster. TBM analysis revealed one small subregion within the cluster that reached peak significance but did not survive the extent threshold.

\section{Temporoparietal pathways}

There were no language-related areas in which subjects with TS had less FA or density than controls. There were, however, regions in the left inferior parietal lobule and the inferior, superior, and middle temporal gyri in which TS subjects exhibited greater FA values than controls. These were also areas in which the TS group exhibited greater white matter density than controls. There were no significant shape differences between the groups within the FA cluster.

\section{Discussion}

The results of this study demonstrate white matter abnormalities in young females with TS. Consistent with known visuospatial and motor deficits in TS, decreases in white matter FA and density were observed in the parieto-occipital, frontoparietal, and sensorimotor pathways. Increases in FA values were found in areas important for language abilities (the inferior parietal and temporal lobe), a relative strength of individuals with TS. Although these regional differences in FA may reflect primary differences in the density, coherence, or myelination of white matter, they also may reflect secondary changes resulting from abnormalities in the gray matter regions they connect. These findings demonstrate that FA can potentially be used to further map networks of brain regions affected in TS.

\section{Frontoparietal and parieto-occipital pathways}

Visuospatial abilities involve a distributed network that includes the parieto-occipital and prefrontal cortices (Burbaud et al., 1999; Menon et al., 2000). Visuospatial dysfunction in TS may relate to our findings of decreased FA in parieto- occipital white matter extending into the frontal lobe; this pathway appeared to correspond to the SLF. The SLF is a prominent white matter structure that extends in an arc above the insula from the frontal lobe to fan out into the parietal, occipital, and temporal lobes. Abnormal white matter within visuospatial pathways of the SLF is potentially consistent with volumetric studies of the parieto-occipital region in TS (Murphy et al., 1993; Reiss et al., 1995; Brown et al., 2002, 2004; Molko et al., 2003). Functional imaging studies have shown that regions located within this pathway contribute to visuospatial processing. Specifically, the inferior parietal lobe contributes to spatial encoding and working memory storage mechanisms, the superior parietal lobe processes spatial orientation cues, and the MFG is involved in working memory, attention, and visuospatial skills (Kesler et al., 2004b). Our findings of reduced white matter anisotropy in the frontoparietal pathway may indicate disrupted connectivity within the visuospatial network of persons with TS and may contribute to functional activation deficits found in these regions (Kesler et al., 2004b).

The prefrontal cortex has been implicated in executive functions underlying visuospatial working memory (Smith and Jonides, 1999). Hence, white matter abnormalities in the right prefrontal lobe may be linked to activation deficits observed during executive functioning tasks in this condition (Haberecht et al., 2001). Often present in TS, deficits in the triad of planning, attentional set shifting, and working memory skills are thought to result from aberrant frontostriatal circuitry (Cummings, 1993; Haberecht et al., 2001). Thus, our white matter findings in the right prefrontal lobe may be related to aberrant frontostriatal circuitry in TS and contribute to the neural basis of executive functioning deficits in this condition.

\section{Temporoparietal pathways}

The finding of increased FA and density in the left inferior parietal lobe extending into the deep white matter of the superior, middle, and inferior temporal gyri are consistent with the hypothesis of preserved or relatively strong verbal ability in TS. This finding also is consistent with our recent report of increased left STG white matter in TS (Kesler et al., 2003). Previous VBM and DTI studies of TS reported increased gray and white matter density and increased FA in the temporooccipito-parietal region (Molko et al., 2003, 2004). Our findings include much of the left hemisphere perisylvian language area, including the temporal lobe and inferior parietal lobule. Females with TS tend to have well developed abilities associated with these regions, including auditory perception, phonological processing skills, word knowledge, verbal comprehension, and verbal reasoning (Temple and Carney, 1993; Romans et al., 1998; Rourke et al., 2002). Increased FA and density in regions associated with these skills suggests enhanced pathway development attributable to preferential or excess use. To compensate for their nonverbal deficits, TS females may rely more on their verbal pathways and thus develop greater anisotropy from increased coherence or density of fibers.

\section{Internal capsule pathways}

This is the first study of TS to report diffuse white matter pathway disruptions extensively throughout the bilateral internal capsule. The convergence of FA and VBM analysis in this region leads to hypotheses about sensorimotor pathways in TS. Our find- 

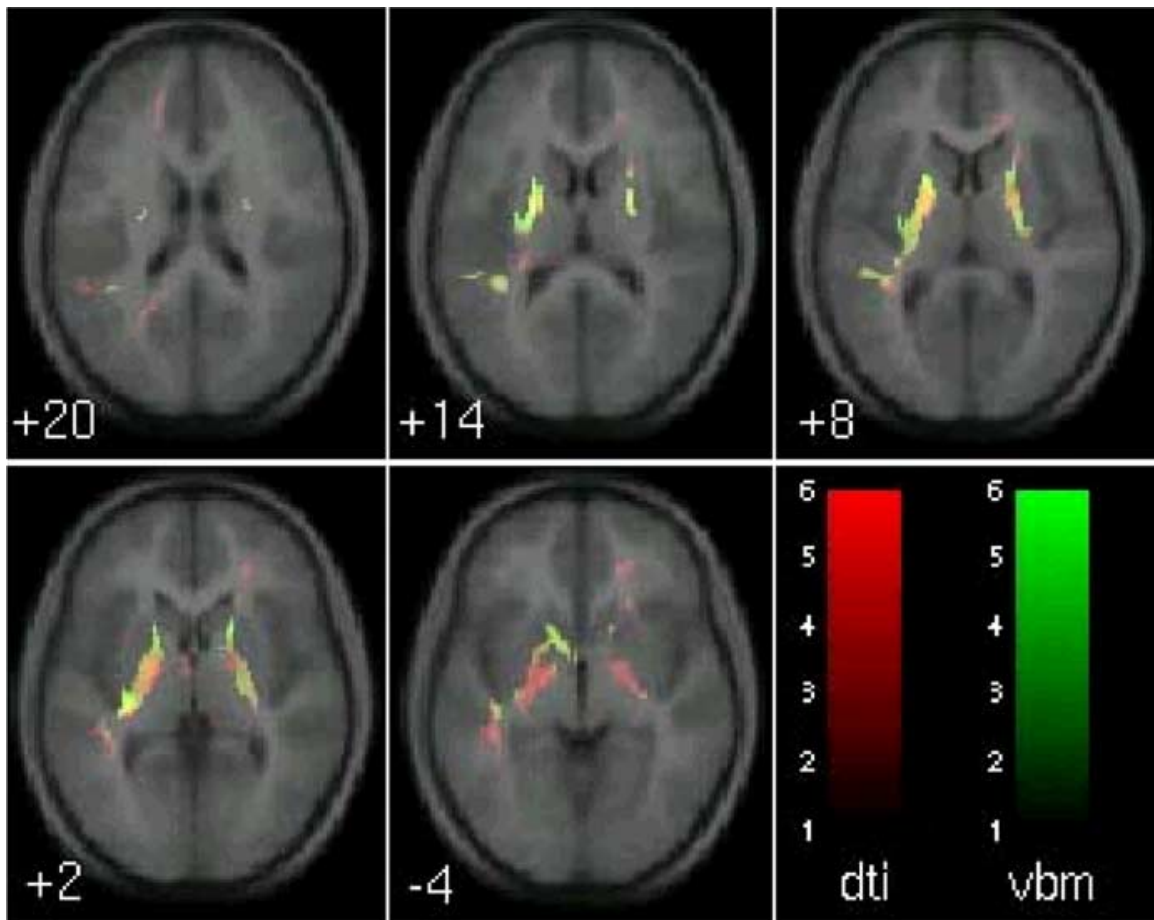

Figure 3. Voxels showing significant reduction in white matter fractional anisotropy in TS are shown in red, and reductions in white matter density are shown in green; overlaps between DTI and VBM findings are shown in yellow. Results through the level of the internal capsule are shown mapped onto an average T1-weighted image of control and TS brains.

ings of decreased density and FA throughout the internal capsule may include sensorimotor neurons from the precentral and postcentral gyri, which project down through the internal capsule, as well as thalamus and basal ganglia. These findings complement gray matter findings of a recent study reporting decreased tissue volume in bilateral postcentral gyri in TS (Brown et al., 2004). Aberrant white matter of the internal capsule may be related to both gross and fine motor dysfunction in TS (Salbenblatt et al., 1989; Nijhuis-van der Sanden et al., 2000). There is some debate as to whether TS individuals perform less accurately than control females on motor tasks attributable to spatial demands (Ross et al., 1996) or motor execution problems independent of visuospatial problems (Nijhuis-van der Sanden et al., 2002). Both FA and VBM findings in the white matter of the internal capsule provide support for the hypothesis that motor executive problems in TS females may have an anatomical basis.

\section{Consistency of TS findings}

A previous DTI study in TS reported increases in FA in the right centrum semiovale and left temporo-occipito-parietal region similar to those reported here (Molko et al., 2004). The coordinate position of the maximum peak in right centrum semiovale near the MFG found by the Molko et al. study was very close to the one identified in this study (Molko et al., 2004, 30, -3, 33; present study, 33, 0, 31). However, there were also differences between the studies. Molko et al. did not find any areas of decreased FA or density in the internal capsule. One possible explanation of the discrepancies between studies is the genomic criteria used for subject selection. Mosaic karyotypes contribute to the " $\mathrm{X}$ chromosome dosage effect" and cause broad heterogeneity in physical and cognitive manifestations of TS (Murphy et al., 1997; Kesler et al., 2003).
The study described here used only monosomic 45X TS females.

Molko et al. suggest that some of their FA results can be attributed to the presence of a gray-white matter boundary shift and not true anisotropy differences. They found shifted central sulci and altered gyrification of the right intraparietal sulcus. We used TBM to detect whether our FA findings were attributable to shape differences or were valid white matter abnormalities. The results for all clusters were negative. However, one small peak was found in the left precentral gyrus but was not significant because of the cluster extent threshold. This finding may relate to the shape differences Molko et al. found in the central sulcus. Although it is possible that our analysis lacks sensitivity because of sample size, it is comparable with the 14 subjects of Molko et al. Similarly, their findings of high variability in gyrification may be attributed to the use of mosaic genotypes (Molko et al., 2003). Additionally, our study contained children, adolescents, and young adults who possess potential developmental differences in motor functioning (Ross et al., 1996) and in white matter structure as a function of age (Klingberg et al., 1999; Schmithorst et al., 2002; Barnea-Goraly et al., 2005).

\section{Limitations}

The strengths of this study include the homogeneous population resulting from including only nonmosaic genotypes. However, limitations include sample size, lack of cognitive data, and variation in the length and dosage of hormone replacement therapies in TS subjects. Variations within the treatment of subjects in our study may alter brain development and thus influence the white matter coherence and density. More specifically, estrogen has been shown to alter neurite growth (Toran-Allerand, 1991), and estrogen therapy was shown to partially reverse some of the neurocognitive and motor deficits observed in TS (Ross et al., 2000).

Another possible limitation stems from the use of a nonisotropic voxel (of $1.9 \times 1.9 \times 6 \mathrm{~mm}$ ) in our analysis, which may increase the risk of partial volume effects. However, these dimensions were chosen on a pragmatic basis, to increase the signal-tonoise ratio within an acceptable scan time for subjects.

Finally, although our study raises important hypotheses and implications for understanding the neural pathophysiology of TS, it is not possible to determine the histologic structure of white matter based solely on imaging findings. Changes in FA can result from changes in fiber diameter, density, myelination, and coherence and changes in extracellular diffusion. It is yet to be determined which of these is affected in TS. In addition, interpretation of results should be performed with caution, because results can differ based on whether the voxel examined lies within parallel fiber bundles or crossing fibers. In voxels containing crossing fibers, diffusion in opposing directions results in an overall reduction in FA compared with voxels containing fiber bundles in the same orientation. Thus, reduction in fiber diameter, density myelin, or fiber-tract coherence within one of the crossing fibers will increase FA values, whereas the same reduction in a voxel 
within a parallel fiber will reduce FA values. Additional studies, using direct visualization of brain tissue, are needed to determine the underlying structure of white matter in TS.

\section{Conclusions}

TS provides a unique opportunity for investigating the linkages among genes, neurobiology, and cognitive development. In TS, the presence of white matter abnormalities in combination with previously demonstrated cortical abnormalities could have direct implications for elucidating the genetic and neurodevelopmental foundations of cognitive variation in TS. The alterations in white matter morphology detected by DTI and confirmed with VBM and TBM point to a neuroanatomical correlate in TS for the depressed spatial/motor ability and relatively strong verbal ability. Our findings suggest the need for more research into how $\mathrm{X}$ chromosome genes play a role in white matter development.

\section{References}

Ashburner J, Friston KJ (2000) Voxel-based morphometry: the methods. NeuroImage 11:805-821.

Barnea-Goraly N, Menon V, Eckert M, Tamm L, Bammer R, Karchemskiy A, Dant CC, Reiss AL (2005) White matter development during childhood and adolescence: a cross-sectional diffusion tensor imaging study. Cereb Cortex 15:1848-1854.

Basser PJ (1995) Inferring microstructural features and the physiological state of tissues from diffusion-weighted images. NMR Biomed 8:333-344.

Basser PJ, Pierpaoli C (1996) Microstructural and physiological features of tissues elucidated by quantitative-diffusion-tensor MRI. J Magn Reson B 111:209-219.

Basser PJ, Mattiello J, LeBihan D (1994) MR diffusion tensor spectroscopy and imaging. Biophys J 66:259-267.

Bender BG, Linden MG, Robinson A (1993) Neuropsychological impairment in 42 adolescents with sex chromosome abnormalities. Am J Med Genet 48:169-173.

Brown WE, Kesler SR, Eliez S, Warsofsky IS, Haberecht M, Patwardhan A, Ross JL, Neely EK, Zeng SM, Yankowitz J, Reiss AL (2002) Brain development in Turner syndrome: a magnetic resonance imaging study. Psychiatry Res 116:187-196.

Brown WE, Kesler SR, Eliez S, Warsofsky IS, Haberecht M, Reiss AL (2004) A volumetric study of parietal lobe subregions in Turner syndrome. Dev Med Child Neurol 46:607-609.

Burbaud P, Camus O, Guehl D, Bioulac B, Caille JM, Allard M (1999) A functional magnetic resonance imaging study of mental subtraction in human subjects. Neurosci Lett 273:195-199.

Clark C, Klonoff H, Hayden M (1990) Regional cerebral glucose metabolism in Turner syndrome. Can J Neurol Sci 17:140-144.

Cummings JL (1993) Frontal-subcortical circuits and human behavior. Arch Neurol 50:873-880.

de Crespigny AJ, Moseley ME (1998) Eddy current induced image warping in diffusion weighted EPI. In: ISMRM 6th Meething, p 661. Sydney: ISMRM.

Good CD, Scahill RI, Fox NC, Ashburner J, Friston KJ, Chan D, Crum WR, Rossor MN, Frackowiak RS (2002) Automatic differentiation of anatomical patterns in the human brain: validation with studies of degenerative dementias. NeuroImage 17:29-46.

Good CD, Lawrence K, Thomas NS, Price CJ, Ashburner J, Friston KJ, Frackowiak RS, Oreland L, Skuse DH (2003) Dosage-sensitive X-linked locus influences the development of amygdala and orbitofrontal cortex, and fear recognition in humans. Brain 126:2431-2446.

Haberecht MF, Menon V, Warsofsky IS, White CD, Dyer-Friedman J, Glover GH, Neely EK, Reiss AL (2001) Functional neuroanatomy of visuospatial working memory in Turner syndrome. Hum Brain Mapp 14:96-107.

Jones DK, Symms MR, Cercignani M, Howard RJ (2005) The effect of filter size on VBM analyses of DT-MRI data. NeuroImage 26:546-554.

Kesler SR, Blasey CM, Brown WE, Yankowitz J, Zeng SM, Bender BG, Reiss AL (2003) Effects of X-monosomy and X-linked imprinting on superior temporal gyrus morphology in Turner syndrome. Biol Psychiatry 54:636-646.

Kesler SR, Garrett A, Bender B, Yankowitz J, Zeng SM, Reiss AL (2004a)
Amygdala and hippocampal volumes in Turner syndrome: a highresolution MRI study of X-monosomy. Neuropsychologia 42:1971-1978.

Kesler SR, Haberecht MF, Menon V, Warsofsky IS, Dyer-Friedman J, Neely EK, Reiss AL (2004b) Functional neuroanatomy of spatial orientation processing in turner syndrome. Cereb Cortex 14:174-180.

Klingberg T, Vaidya CJ, Gabrieli JD, Moseley ME, Hedehus M (1999) Myelination and organization of the frontal white matter in children: a diffusion tensor MRI study. NeuroReport 10:2817-2821.

Lippe B (1991) Turner syndrome. Endocrinol Metab Clin North Am 20:121-152.

McCauley E, Kay T, Ito J, Treder R (1987) The Turner syndrome: cognitive deficits, affective discrimination, and behavior problems. Child Dev 58:464-473.

Menon V, Anagnoson RT, Glover GH, Pfefferbaum A (2000) Basal ganglia involvement in memory-guided movement sequencing. NeuroReport 11:3641-3645.

Molko N, Cachia A, Riviere D, Mangin JF, Bruandet M, Le Bihan D, Cohen L, Dehaene S (2003) Functional and structural alterations of the intraparietal sulcus in a developmental dyscalculia of genetic origin. Neuron 40:847-858.

Molko N, Cachia A, Riviere D, Mangin JF, Bruandet M, LeBihan D, Cohen L, Dehaene S (2004) Brain anatomy in Turner syndrome: evidence for impaired social and spatial-numerical networks. Cereb Cortex 14:840-850.

Moseley I (1991) Imaging in neuro-ophthalmology. Curr Opin Neurol Neurosurg 4:829-832.

Murphy DG, DeCarli C, Daly E, Haxby JV, Allen G, White BJ, McIntosh AR, Powell CM, Horwitz B, Rapoport SI, et al. (1993) X-chromosome effects on female brain: a magnetic resonance imaging study of Turner's syndrome. Lancet 342:1197-1200.

Murphy DG, Allen G, Haxby JV, Largay KA, Daly E, White BJ, Powell CM, Schapiro MB (1994) The effects of sex steroids, and the X chromosome, on female brain function: a study of the neuropsychology of adult Turner syndrome. Neuropsychologia 32:1309-1323.

Murphy DG, Mentis MJ, Pietrini P, Grady C, Daly E, Haxby JV, De La Granja M, Allen G, Largay K, White BJ, Powell CM, Horwitz B, Rapoport SI, Schapiro MB (1997) A PET study of Turner's syndrome: effects of sex steroids and the X chromosome on brain. Biol Psychiatry 41:285-298.

Muzik O, Chugani DC, Juhasz C, Shen C, Chugani HT (2000) Statistical parametric mapping: assessment of application in children. NeuroImage 12:538-549.

Nijhuis-van der Sanden RW, Smits-Engelsman BC, Eling PA (2000) Motor performance in girls with Turner syndrome. Dev Med Child Neurol 42:685-690.

Nijhuis-van der Sanden MW, Smits-Engelsman BC, Eling PA, Nijhuis BJ, Van Galen GP (2002) Low elementary movement speed is associated with poor motor skill in Turner's syndrome. Dev Neuropsychol 22:643-670.

Pennington BF, Heaton RK, Karzmark P, Pendleton MG, Lehman R, Shucard DW (1985) The neuropsychological phenotype in Turner syndrome. Cortex 21:391-404.

Poline JB, Worsley KJ, Evans AC, Friston KJ (1997) Combining spatial extent and peak intensity to test for activations in functional imaging. NeuroImage 5:83-96.

Reiss AL, Mazzocco MM, Greenlaw R, Freund LS, Ross JL (1995) Neurodevelopmental effects of X monosomy: a volumetric imaging study. Ann Neurol 38:731-738.

Romans SM, Stefanatos G, Roeltgen DP, Kushner H, Ross JL (1998) Transition to young adulthood in Ullrich-Turner syndrome: neurodevelopmental changes. Am J Med Genet 79:140-147.

Ross J, Zinn A, McCauley E (2000) Neurodevelopmental and psychosocial aspects of Turner syndrome. Ment Retard Dev Disabil Res Rev 6:135-141.

Ross JL, Kushner H, Roeltgen DP (1996) Developmental changes in motor function in girls with Turner syndrome. Pediatr Neurol 15:317-322.

Rourke BP, Ahmad SA, Collins DW, Hayman-Abello BA, Hayman-Abello SE, Warriner EM (2002) Child clinical/pediatric neuropsychology: some recent advances. Annu Rev Psychol 53:309-339.

Rovet J, Ireland L (1994) Behavioral phenotype in children with Turner syndrome. J Pediatr Psychol 19:779-790.

Salbenblatt JA, Meyers DC, Bender BG, Linden MG, Robinson A (1989) Gross and fine motor development in 45,X and 47,XXX girls. Pediatrics 84:678-682.

Schmithorst VJ, Wilke M, Dardzinski BJ, Holland SK (2002) Correlation of 
white matter diffusivity and anisotropy with age during childhood and adolescence: a cross-sectional diffusion-tensor MR imaging study. Radiology 222:212-218.

Skuse DH, James RS, Bishop DV, Coppin B, Dalton P, Aamodt-Leeper G, Bacarese-Hamilton M, Creswell C, McGurk R, Jacobs PA (1997) Evidence from Turner's syndrome of an imprinted X-linked locus affecting cognitive function. Nature 387:705-708.

Smith EE, Jonides J (1999) Storage and executive processes in the frontal lobes. Science 283:1657-1661.

Tamm L, Menon V, Reiss AL (2003) Abnormal prefrontal cortex function during response inhibition in turner syndrome: functional magnetic resonance imaging evidence. Biol Psychiatry 53:107-111.
Temple CM, Carney RA (1993) Intellectual functioning of children with Turner syndrome: a comparison of behavioural phenotypes. Dev Med Child Neurol 35:691-698.

Toran-Allerand CD (1991) Organotypic culture of the developing cerebral cortex and hypothalamus: relevance to sexual differentiation. Psychoneuroendocrinology 16:7-24.

Waber DP (1979) Neuropsychological aspects of Turner's syndrome. Dev Med Child Neurol 21:58-70.

Zinn AR, Tonk VS, Chen Z, Flejter WL, Gardner HA, Guerra R, Kushner H, Schwartz S, Sybert VP, Van Dyke DL, Ross JL (1998) Evidence for a Turner syndrome locus or loci at Xp11.2-p22.1. Am J Hum Genet 63: 1757-1766. 